\title{
Politique
}

Politique

\section{Louis M. IMBEAU, Donnor Aid - The Determinants of Development Allocations to Third World Countries. A Comparative Analysis, New York, Peter Lang, American University Studies, 1989, 228 p.}

\section{Saâd Amrani}

Numéro 18, automne 1990

URI : https://id.erudit.org/iderudit/040671ar

DOI : https://doi.org/10.7202/040671ar

Aller au sommaire du numéro

Éditeur(s)

Société québécoise de science politique

ISSN

0711-608X (imprimé)

1918-6584 (numérique)

Découvrir la revue

Citer ce compte rendu

Amrani, S. (1990). Compte rendu de [Louis M. IMBEAU, Donnor Aid - The Determinants of Development Allocations to Third World Countries. A

Comparative Analysis, New York, Peter Lang, American University Studies, 1989, 228 p.] Politique, (18), 109-112. https://doi.org/10.7202/040671ar d'utilisation que vous pouvez consulter en ligne.

https://apropos.erudit.org/fr/usagers/politique-dutilisation/ 


\section{Recensions}

Louis M. IMBEAU, Donnor Aid - The Determinants of Development Allocations to Third World Countries. A Comparative Analysis, New York, Peter Lang, American University Studies, 1989, $228 \mathrm{p}$.

Comme le souligne le professeur Robert D. McKinlay dans sa préface de l'ouvrage, Louis Imbeau apporte deux contributions importantes à la recherche sur l'aide au développement. Ainsi présente-t-il une analyse rigoureuse d'un phénomène demeuré jusqu'à maintenant relativement assez peu exploré, soit l'importance inégale accordée à l'aide publique au développement (APD) par les pays donateurs. En effet, la question centrale que pose l'auteur consiste à identifier les facteurs qui expliquent pourquoi l'aide varie considérablement d'un donateur à l'autre (p. 4). Son deuxième apport est de nature théorique. Il tient à la reformulation du modèle de «bounded rationality» de Simon et March pour l'adapter à l'analyse des décisions collectives: les choix gouvernementaux en matière d'aide extérieure.

$\mathrm{La}$ recherche de Louis Imbeau porte sur les transferts au chapitre de l'APD fournie par 17 pays de l'OCDE en 1966, 1971, 1976 et 1981. L'auteur passe en revue, de façon assez sommaire certes, les principales théories de l'aide extérieure pour consacrer l'essentiel de son livre à la vérification empirique des propositions dérivées du modèle de la rationalité collective.

Dans le premier chapitre, il construit son propre cadre théorique où les politiques d'aide sont expliquées en tenant compte 
de l'interrelation entre facteurs objectifs ou environnementaux et facteurs subjectifs. Aussi, ce modèle est fondé sur l'idée centrale voulant que les choix des décideurs sont rationnels, leur objectif ultime étant cependant de retenir la plus satisfaisante et non la meilleure alternative qui s'offre à eux (p. 15).

Le deuxième chapitre est consacré aux définitions de la variable dépendante et des quatre variables explicatives ainsi qu'à la formulation des propositions concernant leur relation. La technique de la régression multiple est utilisée pour déterminer la qualité de la liaison entre les différentes variables dont l'analyse est présentée dans les quatre chapitres suivants (chapitres 3 à 6).

Ainsi, dans le troisième chapitre, l'auteur cherche à savoir dans quelle mesure les transferts d'aide sont déterminés par les intérêts politiques et économiques des pays donateurs. L'analyse statistique montre tout d'abord que l'intérêt manifesté par les pays donateurs à l'égard du Tiers-Monde a été relativement stable (p. 60). Toutefois, cet intérêt s'est accru au milieu de la décennie soixantedix en raison, nous dit l'auteur, de la hausse du prix du brut en 1973 et de la crise pétrolière qui s'ensuivit. Mais plus important encore est le fait que les contributions financières exprimées en fonction du PNB ou des dépenses gouvernementales varient selon l'importance des intérêts économiques et politiques et la conception de l'aide - instrumentaliste versus humanitaire - des donateurs (p. 59). C'est le cas notamment des grandes puissances économiques (États-Unis, Japon) et, dans une moindre mesure, des ex-puissances coloniales européennes présentes un peu partout au Tiers-Monde. D'après les données de l'auteur, le couple intérêt/conception de l'aide représente la variable explicative la plus significative des différences relevées au chapitre de l'aide fournie par les pays de l'OCDE, excepté pour l'année 1966 (pp. 144 et 162). C'est dire qu'en matière d'aide au développement les pays donateurs pensent et agissent d'abord et avant tout en fonction de leurs intérêts.

L'auteur examine également la validité des deux postulats mettant en jeu la perspective «humanitaire» (chapitre 4). Il avance que l'aide au Tiers-Monde est en principe affectée par les besoins des récipiendaires, et que l'impact de ces besoins dépend en fin de 
compte de la conception sous-jacente aux politiques des pays fournisseurs d'aide. Ainsi, les pays plus sensibles aux besoins des pays assistés et ayant une conception plus humanitaire de l'aide devraient consacrer plus d'efforts à ce chapitre, le contraire se produisant lorsque l'aide sert de simple instrument de politique étrangère (conception instrumentaliste). L'analyse statistique ne confirme que très partiellement la validité de ces postulats. Certes, remarque-t-on une plus grande réponse aux besoins des pays en développement depuis la fin des années soixante, que l'auteur attribue à l'enrichissement des pays occidentaux et à l'impact des revendications pour la mise en place d'un nouvel ordre économique international. Cependant, dans de nombreux cas, cette analyse a permis de constater que ce sont les pays les plus «humanitaires» qui contribuent le moins à l'aide au développement. De là la conclusion de l'auteur, qui donne du poids à l'idée largement répandue voulant que l'aide est loin d'être un instrument d'action strictement humanitaire (pp. 88-89). Devant ce paradoxe, l'auteur propose une explication provisoire plutôt intuitive: les contributions relativement moins importantes des pays humanitaires seraient dues au fait qu'ils disposent d'une capacité financière plus limitée (p. 97). Toujours est-il que, de tous les facteurs explicatifs retenus dans le modèle, la variable humanitaire est celle qui est la moins significative lorsque l'on considère le comportement des pays de l'OCDE en matière d'aide, bien que l'auteur ne rejette pas totalement sa validité (p. 158).

Plus loin, Imbeau postule que l'aide varie d'un donateur à l'autre en raison des orientations idéologiques des gouvernements et de l'électorat (chapitre 5). Dans ce sens, il s'attend à ce que les pays où prédomine l'idéologie de "gauche» affectent - si leur capacité financière le permet - une part plus importante du PNB à l'aide au développement que les pays qu'il qualifie de «droite». Ceci parce que les premiers favorisent les politiques égalitaristes assurant une meilleure redistribution des richesses entre le Nord et le Sud (p. 34). De fait, cette hypothèse est confirmée pour toute la période temporelle retenue, à l'exception de l'année 1966. L'auteur croit que cette anomalie résulte du fait que les pays dits de «gau- 
che» n'avaient pas encore mis de l'avant une politique d'aide qui corresponde à leur orientation idéologique (pp. 157-158).

Il n'y a pas lieu de contester ici la qualité de l'ouvrage qui a d'ailleurs valu à son auteur le prix Stein-Rokkan décerné par le Conseil international des sciences sociales (Paris). Louis Imbeau a eu le mérite de présenter une approche théorique qui constitue une contribution sérieuse à l'étude des déterminants de l'aide au développement. Par ailleurs, l'analyse présente un intérêt incontestable tant par sa rigueur méthodologique que par la clarté du raisonnement. Cependant, deux limites doivent en particulier être mentionnées. La première a trait au choix des indicateurs que retient l'auteur pour mesurer certaines variables (idéologie, besoins des récipiendaires de l'aide). D'autre part, l'auteur admet lui-même que son étude soulève plus de questions qu'elle n'en résoud (p. 163). On peut se demander si cela n'est pas attribuable aux démarches qui accordent une trop grande place aux techniques quantitatives pour l'analyse des phénomènes politiques.

Saâd Amrani

Université de Montréal 